PROCEEDINGS OF THE

AMERICAN MATHEMATICAL SOCIETY

Volume 140, Number 1, January 2012, Pages 189-197

S 0002-9939(2011)11123-7

Article electronically published on May 19, 2011

\title{
A UNIQUENESS THEOREM FOR MEROMORPHIC MAPPINGS WITH TWO FAMILIES OF HYPERPLANES
}

\author{
GERD DETHLOFF, SY DUC QUANG, AND TRAN VAN TAN
}

(Communicated by Franc Forstneric)

\begin{abstract}
In this paper, we extend the uniqueness theorem for meromorphic mappings to the case where the family of hyperplanes depends on the meromorphic mapping and where the meromorphic mappings may be degenerate.
\end{abstract}

\section{INTRODUCTION}

The uniqueness problem of meromorphic mappings under a condition on the inverse images of divisors was first studied by Nevanlinna [6]. He showed that for two nonconstant meromorphic functions $f$ and $g$ on the complex plane $\mathbb{C}$, if they have the same inverse images for five distinct values, then $f \equiv g$. In 1975, Fujimoto [3] generalized Nevanlinna's result to the case of meromorphic mappings of $\mathbb{C}^{m}$ into $\mathbb{C} P^{n}$. He showed that for two linearly nondegenerate meromorphic mappings $f$ and $g$ of $\mathbb{C}^{m}$ into $\mathbb{C} P^{n}$, if they have the same inverse images counted with multiplicities for $3 n+2$ hyperplanes in general position in $\mathbb{C} P^{n}$, then $f \equiv g$.

In 1983, Smiley [9] showed that

Theorem 1.1. Let $f, g$ be linearly nondegenerate meromorphic mappings of $\mathbb{C}^{m}$ into $\mathbb{C} P^{n}$. Let $\left\{H_{j}\right\}_{j=1}^{q}(q \geq 3 n+2)$ be hyperplanes in $\mathbb{C} P^{n}$ in general position. Assume that

a) $f^{-1}\left(H_{j}\right)=g^{-1}\left(H_{j}\right)$ for all $1 \leq j \leq q$ (as sets),

b) $\operatorname{dim}\left(f^{-1}\left(H_{i}\right) \cap f^{-1}\left(H_{j}\right)\right) \leq m-2$ for all $1 \leq i<j \leq q$,

c) $f=g$ on $\bigcup_{j=1}^{q} f^{-1}\left(H_{j}\right)$.

Then $f \equiv g$.

In 2006, Thai and Quang [11] generalized this result of Smiley to the case where $q \geq 3 n+1$ and $n \geq 2$. In 2009, Dethloff and Tan [2] showed that for every nonnegative integer $c$ there exists a positive integer $N(c)$ depending only on $c$ such that Theorem 1.1 remains valid if $q \geq 3 n+2-c$ and $n \geq N(c)$. They also showed that the coefficient of $n$ in the formula of $q$ can be replaced by a number which is smaller than 3 for all $n \gg 0$. Furthermore, they established a uniqueness theorem for the case of $2 n+3$ hyperplanes and multiplicities are truncated by $n$. At the same time, they strongly generalized many uniqueness theorems of previous authors such as

Received by the editors November 1, 2010.

2010 Mathematics Subject Classification. Primary 32H30; Secondary 32H04, 30D35.

This work was done during a stay of the third-named author at the Institut des Hautes Études Scientifiques, France. He wishes to express his gratitude to this institute. The second- and the third-named authors are supported by an NAFOSTED grant of Vietnam.

(C)2011 American Mathematical Society Reverts to public domain 28 years from publication 
Fujimoto [4, Ji [5] and Stoll [10. Recently, by using again the technique of Dethloff and Tan 2], Chen and Yan [1] showed that the assumption "multiplicities are truncated by $n$ " in the result of Dethloff and Tan can be replaced by "multiplicities are truncated by 1". In [8], Quang examined the uniqueness problem for the case of $2 n+2$ hyperplanes.

We would like to note that so far, all results on the uniqueness problem have still been restricted to the case where meromorphic mappings are sharing a common family of hyperplanes. The purpose of this paper is to introduce a uniqueness theorem for the case where the family of hyperplanes depends on the meromorphic mapping. We also will allow that the meromorphic mappings may be degenerate. For this purpose we introduce some new techniques which can also be used to obtain simpler proofs for many other uniqueness theorems.

We shall prove the following uniqueness theorem:

Theorem 1.2. Let $f, g$ be nonconstant meromorphic mappings of $\mathbb{C}^{m}$ into $\mathbb{C} P^{n}$. Let $\left\{H_{j}\right\}_{j=1}^{q}$ and $\left\{L_{j}\right\}_{j=1}^{q}(q>2 n+2)$ be families of hyperplanes in $\mathbb{C} P^{n}$ in general position. Assume that

a) $f^{-1}\left(H_{j}\right)=g^{-1}\left(L_{j}\right)$ for all $1 \leq j \leq q$,

b) $\operatorname{dim}\left(f^{-1}\left(H_{i}\right) \cap f^{-1}\left(H_{j}\right)\right) \leq m-2$ for all $1 \leq i<j \leq q$,

c) $\frac{\left(f, H_{i}\right)}{\left(g, L_{i}\right)}=\frac{\left(f, H_{j}\right)}{\left(g, L_{j}\right)}$ on $\bigcup_{k=1}^{q} f^{-1}\left(H_{k}\right) \backslash\left(f^{-1}\left(H_{i}\right) \cup f^{-1}\left(H_{j}\right)\right)$ for all $1 \leq i<$ $j \leq q$.

Then the following assertions hold:

i) $\operatorname{dim}\langle\operatorname{Im} f\rangle=\operatorname{dim}\langle\operatorname{Im} g\rangle \stackrel{\text { Def. }}{=} p$, where for a subset $X \subset \mathbb{C} P^{n}$, we denote by $\langle X\rangle$ the smallest projective subspace of $\mathbb{C} P^{n}$ containing $X$.

ii) If

$$
(*) \quad q>\frac{2 n+3-p+\sqrt{(2 n+3-p)^{2}+8(p-1)(2 n-p+1)}}{2}(\geq 2 n+2),
$$

then

$$
\frac{\left(f, H_{1}\right)}{\left(g, L_{1}\right)} \equiv \cdots \equiv \frac{\left(f, H_{q}\right)}{\left(g, L_{q}\right)} .
$$

Furthermore, there exists a linear projective transformation $\mathcal{L}$ of $\mathbb{C} P^{n}$ into itself such that $\mathcal{L}(f) \equiv g$ and $\mathcal{L}\left(H_{j} \cap\langle\operatorname{Im} f\rangle\right)=L_{j} \cap \mathcal{L}(\langle\operatorname{Im} f\rangle)$ for all $j \in\{1, \ldots, q\}$.

Remark. 1) In Theorem 1.2, condition $c$ ) is well defined since, by condition $a$ ), $\frac{\left(f, H_{i}\right)}{\left(g, L_{i}\right)}$ is a (nonvanishing) holomorphic function outside $f^{-1}\left(H_{i}\right)$.

$2)$ The condition $(*)$ is satisfied in the following cases:

+) $q \geq 2 n+3$ and $p \in\{1,2, n-1, n\}, n \in \mathbb{Z}^{+}$.

+) $q \geq 2 n+p+1$ and $p \in\{2,3, \ldots, n\}, n \in \mathbb{Z}^{+}$.

3) If there exists a subset $\left\{j_{0}, \ldots, j_{n}\right\} \subset\{1, \ldots, q\}$ such that $H_{j_{i}} \equiv L_{j_{i}}$ for all $i \in\{0, \ldots, n\}$, then the proof of Theorem 1.2 implies that $f \equiv g$.

4) For the special case where $f, g$ are linearly nondegenerate (i.e., $p=n$ ) and $H_{j} \equiv L_{j}$, from Theorem 1.2 we get again the results of Dethloff and Tan [2] and Chen and Yan [1].

\section{Preliminaries}

We set $\|z\|:=\left(\left|z_{1}\right|^{2}+\cdots+\left|z_{m}\right|^{2}\right)^{1 / 2}$ for $z=\left(z_{1}, \ldots, z_{m}\right) \in \mathbb{C}^{m}$ and define

$$
B(r):=\left\{z \in \mathbb{C}^{m}:\|z\|<r\right\}, \quad S(r):=\left\{z \in \mathbb{C}^{m}:\|z\|=r\right\}
$$


for all $0<r<\infty$. Define

$$
\begin{aligned}
d^{c} & :=\frac{\sqrt{-1}}{4 \pi}(\bar{\partial}-\partial), \quad v:=\left(d d^{c}\|z\|^{2}\right)^{m-1}, \\
\sigma & :=d^{c} \log \|z\|^{2} \wedge\left(d d^{c} \log \|z\|^{2}\right)^{m-1} .
\end{aligned}
$$

Let $F$ be a nonzero holomorphic function on $\mathbb{C}^{m}$. For each $a \in \mathbb{C}^{m}$, expanding $F$ as $F=\sum P_{i}(z-a)$ with homogeneous polynomials $P_{i}$ of degree $i$ around $a$, we define

$$
\nu_{F}(a):=\min \left\{i: P_{i} \not \equiv 0\right\} .
$$

Let $\varphi$ be a nonzero meromorphic function on $\mathbb{C}^{m}$. We define the zero divisor $\nu_{\varphi}$ as follows: For each $z \in \mathbb{C}^{m}$, we choose nonzero holomorphic functions $F$ and $G$ on a neighborhood $U$ of $z$ such that $\varphi=F / G$ on $U$ and $\operatorname{dim}\left(F^{-1}(0) \cap G^{-1}(0)\right) \leq m-2$. Then we put $\nu_{\varphi}(z):=\nu_{F}(z)$.

Let $\nu$ be a divisor in $\mathbb{C}^{m}$ and let $k$ be a positive integer or $+\infty$. Set $|\nu|:=$ $\overline{\{z: \nu(z) \neq 0\}}$ and $\nu^{[k]}(z):=\min \{\nu(z), k\}$.

The truncated counting function of $\nu$ is defined by

$$
N^{[k]}(r, \nu):=\int_{1}^{r} \frac{n^{[k]}(t)}{t^{2 m-1}} d t \quad(1<r<+\infty),
$$

where

$$
n^{[k]}(t)= \begin{cases}\int_{|\nu| \cap B(t)} \nu^{[k]} \cdot v & \text { for } m \geq 2, \\ \sum_{|z| \leq t} \nu^{[k]}(z) & \text { for } m=1 .\end{cases}
$$

We simply write $N(r, \nu)$ for $N^{[+\infty]}(r, \nu)$.

For a nonzero meromorphic function $\varphi$ on $\mathbb{C}^{m}$, we set $N_{\varphi}^{[k]}(r):=N^{[k]}\left(r, \nu_{\varphi}\right)$ and $N_{\varphi}(r):=N^{[+\infty]}\left(r, \nu_{\varphi}\right)$. We have the following Jensen's formula:

$$
N_{\varphi}(r)-N_{\frac{1}{\varphi}}(r)=\int_{S(r)} \log |\varphi| \sigma-\int_{S(1)} \log |\varphi| \sigma .
$$

Let $f: \mathbb{C}^{m} \longrightarrow \mathbb{C} P^{n}$ be a meromorphic mapping. For an arbitrary fixed homogeneous coordinate system $\left(w_{0}: \cdots: w_{n}\right)$ in $\mathbb{C} P^{n}$, we take a reduced representation $f=\left(f_{0}: \cdots: f_{n}\right)$, which means that each $f_{i}$ is a holomorphic function on $\mathbb{C}^{m}$ and $f(z)=\left(f_{0}(z): \cdots: f_{n}(z)\right)$ outside the analytic set $\left\{f_{0}=\cdots=f_{n}=0\right\}$ of codimension $\geq 2$. Set $\|f\|=\left(\left|f_{0}\right|^{2}+\cdots+\left|f_{n}\right|^{2}\right)^{1 / 2}$. The characteristic function $T_{f}(r)$ of $f$ is defined by

$$
T_{f}(r):=\int_{S(r)} \log \|f\| \sigma-\int_{S(1)} \log \|f\| \sigma, \quad 1<r<+\infty .
$$

For a meromorphic function $\varphi$ on $\mathbb{C}^{m}$, the characteristic function $T_{\varphi}(r)$ of $\varphi$ is defined by considering $\varphi$ as a meromorphic mapping of $\mathbb{C}^{m}$ into $\mathbb{C} P^{1}$.

We state the First Main Theorem and the Second Main Theorem in Value Distribution Theory: For a hyperplane $H: a_{0} w_{0}+\cdots+a_{n} w_{n}=0$ in $\mathbb{C} P^{n}$ with $\operatorname{Im} f \nsubseteq H$, we put $(f, H)=a_{0} f_{0}+\cdots+a_{n} f_{n}$, where $\left(f_{0}: \cdots: f_{n}\right)$ is a reduced representation of $f$. 
First Main Theorem. Let $f$ be a meromorphic mapping of $\mathbb{C}^{m}$ into $\mathbb{C} P^{n}$, and $H$ be a hyperplane in $\mathbb{C} P^{n}$ such that $(f, H) \not \equiv 0$. Then

$$
N_{(f, H)}(r) \leq T_{f}(r)+O(1) \text { for all } r>1 .
$$

Let $n, N, q$ be positive integers with $q \geq 2 N-n+1$ and $N \geq n$. We say that hyperplanes $H_{1}, \ldots, H_{q}$ in $\mathbb{C} P^{n}$ are in $N$-subgeneral position if $\bigcap_{i=0}^{N} H_{j_{i}}=\emptyset$ for every subset $\left\{j_{0}, \ldots, j_{N}\right\} \subset\{1, \ldots, q\}$.

Cartan-Nochka Second Main Theorem ([7, Theorem 3.1). Let $f$ be a linearly nondegenerate meromorphic mapping of $\mathbb{C}^{m}$ into $\mathbb{C} P^{n}$ and $H_{1}, \ldots, H_{q}$ hyperplanes in $\mathbb{C} P^{n}$ in $N$-subgeneral position $(q \geq 2 N-n+1)$. Then

$$
(q-2 N+n-1) T_{f}(r) \leq \sum_{j=1}^{q} N_{\left(f, H_{j}\right)}^{[n]}(r)+o\left(T_{f}(r)\right)
$$

for all $r$ except for a subset $E$ of $(1,+\infty)$ of finite Lebesgue measure.

\section{Proof of Theorem 1.2}

We first remark that $f^{-1}\left(H_{j}\right)=g^{-1}\left(L_{j}\right) \neq \mathbb{C}^{m}$ for all $j \in\{1, \ldots, q\}$, and that therefore $\left\{H_{j} \cap\langle\operatorname{Im} f\rangle\right\}_{j=1}^{q}$ (respectively $\left\{L_{j} \cap\langle\operatorname{Im} g\rangle\right\}_{j=1}^{q}$ ) are hyperplanes in $\langle\operatorname{Im} f\rangle$ (respectively $\langle\operatorname{Im} g\rangle$ ) in $n$-subgeneral position: Indeed, otherwise there exists $t \in\{1, \ldots, q\}$ such that $f^{-1}\left(H_{t}\right)=\mathbb{C}^{m}$. Then by the assumption $b$ ) we have $\operatorname{dim} f^{-1}\left(H_{j}\right) \leq m-2$ for all $j \in\{1, \ldots, q\} \backslash\{t\}$. Therefore, $f^{-1}\left(H_{j}\right)=\emptyset$ for all $j \in\{1, \ldots, q\} \backslash\{t\}$. Then $\langle\operatorname{Im} f\rangle \not \subset H_{j}$ for all $j \in\{1, \ldots, q\} \backslash\{t\}$. Thus, $\left\{H_{j} \cap\langle\operatorname{Im} f\rangle\right\}_{\substack{j=1 \\ j \neq t}}^{q}$ are hyperplanes in $\langle\operatorname{Im} f\rangle$ in $n$-subgeneral position.

By the Cartan-Nochka Second Main Theorem, we have

$$
(q-2 n+\operatorname{dim}\langle\operatorname{Im} f\rangle-2) T_{f}(r) \leq \sum_{\substack{j=1 \\ j \neq t}}^{q} N_{\left(f, H_{j}\right)}^{[\operatorname{dim}\langle\operatorname{Im} f\rangle]}(r)+o\left(T_{f}(r)\right)=o\left(T_{f}(r)\right) .
$$

This is a contradiction to the fact that $q>2 n+2$.

Since $\left\{H_{j}\right\}_{j=1}^{n+1}$ and $\left\{L_{j}\right\}_{j=1}^{n+1}$ are families of hyperplanes in general position, $\tilde{f}:=\left(\left(f, H_{1}\right): \cdots:\left(f, H_{n+1}\right)\right)$ and $\tilde{g}:=\left(\left(g, L_{1}\right): \cdots:\left(g, L_{n+1}\right)\right)$ are reduced representations of meromorphic mappings $\tilde{f}$ and $\tilde{g}$ respectively of $\mathbb{C}^{m}$ into $\mathbb{C} P^{n}$. Furthermore, $\operatorname{dim}\langle\operatorname{Im} f\rangle=\operatorname{dim}\langle\operatorname{Im} \tilde{f}\rangle, \operatorname{dim}\langle\operatorname{Im} g\rangle=\operatorname{dim}\langle\operatorname{Im} \tilde{g}\rangle, T_{\tilde{f}}(r)=T_{f}(r)+O(1)$ and $T_{\tilde{g}}(r)=T_{g}(r)+O(1)$.

By assumptions $a$ ) and $c$ ) we have that

$$
\tilde{f}=\tilde{g} \quad \text { on } \bigcup_{j=1}^{q} f^{-1}\left(H_{j}\right) .
$$

We now prove that

$$
\operatorname{dim}\langle\operatorname{Im} f\rangle=\operatorname{dim}\langle\operatorname{Im} g\rangle \stackrel{\text { Def. }}{=} p .
$$

This is equivalent to proving that $\operatorname{dim}\langle\operatorname{Im} \tilde{f}\rangle=\operatorname{dim}\langle\operatorname{Im} \tilde{g}\rangle$. Therefore, it suffices to show that for any hyperplane $H$ in $\mathbb{C} P^{n}$, then

$$
(H, \tilde{f}) \equiv 0 \quad \text { if and only if } \quad(H, \tilde{g}) \equiv 0 .
$$


Suppose that the above assertion does not hold. Without loss of the generality, we may assume that there exists a hyperplane $H$ such that $(H, \tilde{f}) \not \equiv 0$ and $(H, \tilde{g}) \equiv 0$. Then by (3.1) we have

$$
(\tilde{f}, H)=0 \quad \text { on } \bigcup_{j=1}^{q} f^{-1}\left(H_{j}\right) .
$$

By (3.3) and by the First Main Theorem and the Cartan-Nochka Second Main Theorem we have

$$
\begin{aligned}
(q-2 n+\operatorname{dim}\langle\operatorname{Im} f\rangle-1) T_{f}(r)+O(1) & \leq \sum_{j=1}^{q} N_{\left(f, H_{j}\right)}^{[\operatorname{dim}\langle\operatorname{Im} f\rangle]}(r)+o\left(T_{f}(r)\right) \\
& \leq \operatorname{dim}\langle\operatorname{Im} f\rangle \sum_{j=1}^{q} N_{\left(f, H_{j}\right)}^{[1]}(r)+o\left(T_{f}(r)\right) \\
& \frac{3.3}{\leq} \operatorname{dim}\langle\operatorname{Im} f\rangle N_{(\tilde{f}, H)}(r)+o\left(T_{f}(r)\right) \\
& \leq \operatorname{dim}\langle\operatorname{Im} f\rangle T_{\tilde{f}}(r)+o\left(T_{f}(r)\right) \\
& =\operatorname{dim}\langle\operatorname{Im} f\rangle T_{f}(r)+o\left(T_{f}(r)\right) .
\end{aligned}
$$

This is a contradiction to the fact that $q>2 n+2$. We complete the proof of (3.2).

Now we prove that

$$
\frac{\left(f, H_{1}\right)}{\left(g, L_{1}\right)} \equiv \cdots \equiv \frac{\left(f, H_{q}\right)}{\left(g, L_{q}\right)}
$$

We distinguish the following two cases:

Case 1. There exists a subset $J:=\left\{j_{0}, \ldots, j_{n}\right\} \subset\{1, \ldots, q\}$ such that

$$
\frac{\left(f, H_{j_{0}}\right)}{\left(g, L_{j_{0}}\right)} \equiv \cdots \equiv \frac{\left(f, H_{j_{n}}\right)}{\left(g, L_{j_{n}}\right)} \stackrel{\text { Def. }}{\equiv} u \text {. }
$$

We have Pole $(u) \cup \operatorname{Zero}(u) \subset f^{-1}\left(H_{j_{0}}\right) \cap f^{-1}\left(H_{j_{1}}\right)$, which is an analytic set of codimension at least 2 by assumption $b)$. Hence, $\operatorname{Pole}(u) \cup \operatorname{Zero}(u)=\emptyset$.

Since $H_{j_{0}}, \ldots, H_{j_{n}}$ are hyperplanes in general position, $F:=\left(\left(f, H_{j_{0}}\right): \cdots\right.$ : $\left.\left(f, H_{j_{n}}\right)\right)$ is the reduced representation of a meromorphic mapping $F$ of $\mathbb{C}^{m}$ into $\mathbb{C} P^{n}$. Still by the same reason $T_{F}(r)=T_{f}(r)+O(1)$.

Suppose that (3.4) does not hold. Then, there exists $i_{0} \in\{1, \ldots, q\} \backslash\left\{j_{0}, \ldots, j_{n}\right\}$ such that

$$
\frac{\left(f, H_{i_{0}}\right)}{\left(g, L_{i_{0}}\right)} \not \equiv u
$$

Since the families $\left\{H_{j}\right\}_{j=1}^{q}$ and $\left\{L_{j}\right\}_{j=1}^{q}$ are in general position, there exist hyperplanes $H^{i_{0}}: a_{0} \omega_{0}+\cdots+a_{n} \omega_{n}=0, L^{i_{0}}: b_{0} \omega_{0}+\cdots+b_{n} \omega_{n}=0$ in $\mathbb{C} P^{n}$ such that $\left(f, H_{i_{0}}\right) \equiv\left(F, H^{i_{0}}\right)$, and $\left(g, L_{i_{0}}\right) \equiv b_{0}\left(g, L_{j_{0}}\right)+\cdots+b_{n}\left(g, L_{j_{n}}\right) \equiv \frac{\left(F, L^{i_{0}}\right)}{u}$. Therefore, by (3.5) we have

$$
\frac{\left(F, H^{i_{0}}\right)}{\left(F, L^{i_{0}}\right)} \equiv \frac{\left(f, H_{i_{0}}\right)}{u\left(g, L_{i_{0}}\right)} \not \equiv 1
$$


By assumption $c$ ) and since $\operatorname{Pole}(u) \cup \operatorname{Zero}(u)=\emptyset$, we have $u=\frac{\left(f, H_{j_{0}}\right)}{\left(g, L_{j_{0}}\right)}=\frac{\left(f, H_{i_{0}}\right)}{\left(g, L_{i_{0}}\right)}=$ $u \frac{\left(F, H^{i_{0}}\right)}{\left(F, L^{i} 0\right)}$ on $\left(\bigcup_{k=1}^{q} f^{-1}\left(H_{k}\right)\right) \backslash\left(f^{-1}\left(H_{i_{0}}\right) \cup f^{-1}\left(H_{j_{0}}\right)\right)$ and $u=\frac{\left(f, H_{j_{1}}\right)}{\left(g, L_{j_{1}}\right)}=\frac{\left(f, H_{i_{0}}\right)}{\left(g, L_{i_{0}}\right)}=$ $u \frac{\left(F, H^{i_{0}}\right)}{\left(F, L^{i_{0}}\right)}$ on $\left(\bigcup_{k=1}^{q} f^{-1}\left(H_{k}\right)\right) \backslash\left(f^{-1}\left(H_{i_{0}}\right) \cup f^{-1}\left(H_{j_{1}}\right)\right)$. Then $\frac{\left(F, H^{i_{0}}\right)}{\left(F, L^{i_{0}}\right)}=1$ on $\left(\bigcup_{k=1}^{q} f^{-1}\left(H_{k}\right)\right) \backslash f^{-1}\left(H_{i_{0}}\right)$.

Therefore,

$$
\begin{aligned}
\sum_{k=1, k \neq i_{0}}^{q} N_{\left(f, H_{k}\right)}^{[1]}(r) & \leq N_{\frac{\left(F, H^{i} 0\right)}{\left(F, L^{i} 0\right)}-1}(r) \\
& \leq T_{\frac{\left(F, H^{i} 0\right)}{\left(F, L^{i} 0\right)}}(r)+O(1) \leq T_{F}(r)+O(1)=T_{f}(r)+O(1) .
\end{aligned}
$$

Therefore, by the Cartan-Nochka Second Main Theorem we have

$$
\begin{array}{r}
T_{f}(r)+O(1) \geq \sum_{k=1, k \neq i_{0}}^{q} N_{\left(f, H_{k}\right)}^{[1]}(r) \geq \sum_{k=1, k \neq i_{0}}^{q} \frac{1}{p} N_{\left(f, H_{k}\right)}^{[p]}(r) \\
\geq \frac{q-2 n+p-2}{p} T_{f}(r)-o\left(T_{f}(r)\right) .
\end{array}
$$

This implies that $q \leq 2 n+2$. This is a contradiction. Hence, we get (3.4) in this case.

Case 2. For any subset $J \subset\{1, \ldots, q\}$ with $\# J=n+1$, there exists a pair $i, j \in J$ such that

$$
\frac{\left(f, H_{i}\right)}{\left(g, L_{i}\right)} \not \equiv \frac{\left(f, H_{j}\right)}{\left(g, L_{j}\right)}
$$

We introduce an equivalence relation on $L:=\{1, \cdots, q\}$ as follows: $i \sim j$ if and only if

$$
\operatorname{det}\left(\begin{array}{ll}
\left(f, H_{i}\right) & \left(f, H_{j}\right) \\
\left(g, L_{i}\right) & \left(g, L_{j}\right)
\end{array}\right) \equiv 0 .
$$

Set $\left\{L_{1}, \cdots, L_{s}\right\}=L / \sim$. By the hypothesis of this case, $\# L_{k} \leq n$ for all $k \in$ $\{1, \cdots, s\}$. Without loss of generality, we may assume that $L_{k}:=\left\{i_{k-1}+1, \cdots, i_{k}\right\}$ $(k \in\{1, \cdots, s\})$ where $0=i_{0}<\cdots<i_{s}=q$.

We define the map $\sigma:\{1, \cdots, q\} \rightarrow\{1, \cdots, q\}$ by

$$
\sigma(i)= \begin{cases}i+n & \text { if } i+n \leq q \\ i+n-q & \text { if } i+n>q\end{cases}
$$

It is easy to see that $\sigma$ is bijective and $|\sigma(i)-i| \geq n$ (note that $q>2 n+2$ ). This implies that $i$ and $\sigma(i)$ belong to distinct sets of $\left\{L_{1}, \cdots, L_{s}\right\}$. This implies that for all $i \in\{1, \ldots, q\}$,

$$
P_{i}:=\operatorname{det}\left(\begin{array}{ll}
\left(f, H_{i}\right) & \left(f, H_{\sigma(i)}\right) \\
\left(g, L_{i}\right) & \left(g, L_{\sigma(i)}\right)
\end{array}\right) \not \equiv 0 .
$$

By the assumption and by the definition of function $P_{i}$, we have

$$
\nu_{P_{i}} \geq \min \left\{\nu_{\left(f, H_{i}\right)}, \nu_{\left(g, L_{i}\right)}\right\}+\min \left\{\nu_{\left(f, H_{\sigma(i)}\right)}, \nu_{\left(g, L_{\sigma(i)}\right)}\right\}+\sum_{\substack{j=1 \\ j \neq i, \sigma(i)}}^{q} \nu_{\left(f, H_{j}\right)}^{[1]}
$$

outside an analytic set of codimension $\geq 2$. 
On the other hand, since $f^{-1}\left(H_{k}\right)=g^{-1}\left(L_{k}\right)$ we have

$$
\begin{aligned}
\min \left\{\nu_{\left(f, H_{k}\right)}, \nu_{\left(g, L_{k}\right)}\right\} & \geq \min \left\{\nu_{\left(f, H_{k}\right)}, p\right\}+\min \left\{\nu_{\left(g, L_{k}\right)}, p\right\}-p \min \left\{\nu_{\left(f, H_{k}\right)}, 1\right\} \\
& =\nu_{\left(f, H_{k}\right)}^{[p]}+\nu_{\left(g, L_{k}\right)}^{[p]}-p \nu_{\left(f, H_{k}\right)}^{[1]}
\end{aligned}
$$

for $k \in\{i, \sigma(i)\}$.

Therefore, by (3.6) we have

$$
\begin{aligned}
\nu_{P_{i}} \geq \nu_{\left(f, H_{i}\right)}^{[p]} & +\nu_{\left(g, L_{i}\right)}^{[p]}+\nu_{\left(f, H_{\sigma(i)}\right)}^{[p]}+\nu_{\left(g, L_{\sigma(i)}\right)}^{[p]} \\
& -p \nu_{\left(f, H_{i}\right)}^{[1]}-p \nu_{\left(f, H_{\sigma(i)}\right)}^{[1]}+\sum_{\substack{j=1 \\
j \neq i, \sigma(i)}}^{q} \nu_{\left(f, H_{j}\right)}^{[1]}
\end{aligned}
$$

outside an analytic set of codimension $\geq 2$.

Then for all $i \in\{1, \ldots, q\}$ we have

$$
\begin{aligned}
N_{P_{i}}(r) \geq N_{\left(f, H_{i}\right)}^{[p]}(r) & +N_{\left(g, L_{i}\right)}^{[p]}(r)+N_{\left(f, H_{\sigma(i)}\right)}^{[p]}(r)+N_{\left(g, L_{\sigma(i)}\right)}^{[p]}(r) \\
& -p N_{\left(f, H_{i}\right)}^{[1]}(r)-p N_{\left(f, H_{\sigma(i)}\right)}^{[1]}(r)+\sum_{\substack{j=1 \\
j \neq i, \sigma(i)}}^{q} N_{\left(f, H_{j}\right)}^{[1]}(r) .
\end{aligned}
$$

On the other hand, by Jensen's formula,

$$
\begin{aligned}
N_{P_{i}}(r)= & \int_{S(r)} \log \left|P_{i}\right| \sigma+O(1) \\
\leq & \int_{S(r)} \log \left(\left|\left(f, H_{i}\right)\right|^{2}+\left|\left(f, H_{\sigma(i)}\right)\right|^{2}\right)^{\frac{1}{2}} \sigma \\
& \quad+\int_{S(r)} \log \left(\left|\left(g, L_{i}\right)\right|^{2}+\left|\left(g, L_{\sigma(i)}\right)\right|^{2}\right)^{\frac{1}{2}} \sigma+O(1) \\
\leq & T_{f}(r)+T_{g}(r)+O(1) .
\end{aligned}
$$

Therefore, by (3.7) for all $i \in\{1, \ldots, q\}$ we have

$$
\begin{aligned}
N_{\left(f, H_{i}\right)}^{[p]}(r) & +N_{\left(g, L_{i}\right)}^{[p]}(r)+N_{\left(f, H_{\sigma(i)}\right)}^{[p]}(r)+N_{\left(g, L_{\sigma(i)}\right)}^{[p]}(r) \\
& -p N_{\left(f, H_{i}\right)}^{[1]}(r)-p N_{\left(f, H_{\sigma(i)}\right)}^{[1]}(r)+\sum_{\substack{j=1 \\
j \neq i, \sigma(i)}}^{q} N_{\left(f, H_{j}\right)}^{[1]}(r) \\
& \leq T_{f}(r)+T_{g}(r)+O(1) .
\end{aligned}
$$

By summing up both sides of the above inequality for all $i \in\{1, \ldots, q\}$, we have

$$
\begin{aligned}
2 \sum_{j=1}^{q}\left(N_{\left(f, H_{j}\right)}^{[p]}(r)+N_{\left(g, L_{j}\right)}^{[p]}(r)\right) & +(q-2 p-2) \sum_{j=1}^{q} N_{\left(f, H_{j}\right)}^{[1]}(r) \\
& \leq q\left(T_{f}(r)+T_{g}(r)\right)+O(1) .
\end{aligned}
$$

Therefore, since $f^{-1}\left(H_{j}\right)=g^{-1}\left(L_{j}\right)$ we have

$$
\begin{aligned}
2 \sum_{j=1}^{q}\left(N_{\left(f, H_{j}\right)}^{[p]}(r)+N_{\left(g, L_{j}\right)}^{[p]}(r)\right) & +\frac{q-2 p-2}{2} \sum_{j=1}^{q}\left(N_{\left(f, H_{j}\right)}^{[1]}(r)+N_{\left(g, L_{j}\right)}^{[1]}(r)\right) \\
& \leq q\left(T_{f}(r)+T_{g}(r)\right)+O(1) .
\end{aligned}
$$


Then

$$
\left(2+\frac{q-2 p-2}{2 p}\right) \sum_{j=1}^{q}\left(N_{\left(f, H_{j}\right)}^{[p]}(r)+N_{\left(g, L_{j}\right)}^{[p]}(r)\right) \leq q\left(T_{f}(r)+T_{g}(r)\right)+O(1) .
$$

By (3.11) and by the Cartan-Nochka Second Main Theorem we have

$$
\begin{aligned}
\frac{(q+2 p-2)(q-2 n+p-1)}{2 p} & \left(T_{f}(r)+T_{g}(r)\right) \\
\leq & q\left(T_{f}(r)+T_{g}(r)\right)+o\left(T_{f}(r)+T_{g}(r)\right) .
\end{aligned}
$$

It follows that $(q+2 p-2)(q-2 n+p-1) \leq 2 p q$. Then $q^{2}-(2 n+3-p) q-$ $2(p-1)(2 n+1-p) \leq 0$. This is a contradiction to condition $(*)$ of Theorem 1.2 . Thus we have completed the proof of (3.4).

Assume that $H_{j}: a_{j 0} \omega_{0}+\cdots+a_{j n} \omega_{n}=0$ and $L_{j}: b_{j 0} \omega_{0}+\cdots+b_{j n} \omega_{n}=0 \quad(j=$ $1, \ldots, q)$.

Set

$$
A:=\left(\begin{array}{ccc}
a_{10} & \ldots & a_{1 n} \\
a_{20} & \ldots & a_{2 n} \\
\vdots & \ddots & \vdots \\
a_{(n+1) 0} & \ldots & a_{(n+1) n}
\end{array}\right), B:=\left(\begin{array}{ccc}
b_{10} & \ldots & b_{1 n} \\
b_{20} & \ldots & b_{2 n} \\
\vdots & \ddots & \vdots \\
b_{(n+1) 0} & \ldots & b_{(n+1) n}
\end{array}\right) \text {, and } \mathcal{L}=B^{-1} \cdot A .
$$

By (3.4), we have $A(f) \equiv B(g)$, so we get $\mathcal{L}(f) \equiv g$.

Set $H_{j}^{*}=\left(a_{j 0}, \ldots, a_{j n}\right) \in \mathbb{C}^{n+1}, L_{j}^{*}=\left(b_{j 0}, \ldots, b_{j n}\right) \in \mathbb{C}^{n+1}$. We write $H_{j}^{*}=$ $\alpha_{j 1} H_{1}^{*}+\cdots+\alpha_{j(n+1)} H_{n+1}^{*}$ and $L_{j}^{*}=\beta_{j 1} L_{1}^{*}+\cdots+\beta_{j(n+1)} L_{n+1}^{*}$.

By (3.4) we have

$$
\frac{\alpha_{j 1}\left(f, H_{1}\right)+\cdots+\alpha_{j(n+1)}\left(f, H_{n+1}\right)}{\beta_{j 1}\left(g, L_{1}\right)+\cdots+\beta_{j(n+1)}\left(g, L_{n+1}\right)} \equiv \frac{\left(f, H_{1}\right)}{\left(g, L_{1}\right)} \equiv \cdots \equiv \frac{\left(f, H_{n+1}\right)}{\left(g, L_{n+1}\right)}
$$

for all $j \in\{1, \ldots, q\}$.

This implies that

$$
\left(\alpha_{j 1}-\beta_{j 1}\right)\left(f, H_{1}\right)+\cdots+\left(\alpha_{j(n+1)}-\beta_{j(n+1)}\right)\left(f, H_{n+1}\right) \equiv 0
$$

for all $j \in\{1, \ldots, q\}$.

On the other hand, $f: \mathbb{C}^{m} \longrightarrow\langle\operatorname{Im} f\rangle$ is linearly nondegenerate and $\left\{H_{j}\right\}_{j=1}^{n+1}$ are in general position in $\mathbb{C} P^{n}$. Thus, by (3.12) we have

$$
\left(\alpha_{j 1}-\beta_{j 1}\right)\left(\omega, H_{1}\right)+\cdots+\left(\alpha_{j(n+1)}-\beta_{j(n+1)}\right)\left(\omega, H_{n+1}\right)=0
$$

for all $\omega \in\langle\operatorname{Im} f\rangle$ for all $j \in\{1, \ldots, q\}$.

Let the hyperplanes $\alpha_{j}: \alpha_{j 1} \omega_{0}+\cdots+\alpha_{j(n+1)} \omega_{n}=0$ and $\beta_{j}: \beta_{j 1} \omega_{0}+\cdots+$ $\beta_{j(n+1)} \omega_{n}=0 \quad(j=1, \ldots, q)$.

By 3.13) we have

$$
\left(A(\omega), \alpha_{j}\right)=\left(A(\omega), \beta_{j}\right)
$$

for all $\omega \in\langle\operatorname{Im} f\rangle$ and $j \in\{1, \ldots, q\}$. 
For any $j \in\{1, \ldots, q\}$ and for any $\omega \in\langle\operatorname{Im} f\rangle$ we have

$$
\begin{aligned}
\left(\omega, H_{j}\right) & =\alpha_{j 1}\left(\omega, H_{1}\right)+\cdots+\alpha_{j(n+1)}\left(\omega, H_{n+1}\right) \\
& =\left(A(\omega), \alpha_{j}\right) \\
& \stackrel{3.14}{=}\left(A(\omega), \beta_{j}\right) \\
& =\left(B \cdot \mathcal{L}(\omega), \beta_{j}\right) \\
& =\beta_{j 1}\left(\mathcal{L}(\omega), L_{1}\right)+\cdots+\beta_{j(n+1)}\left(\mathcal{L}(\omega), L_{n+1}\right) \\
& =\left(\mathcal{L}(\omega), L_{j}\right) .
\end{aligned}
$$

This implies that $\mathcal{L}\left(\langle\operatorname{Im} f\rangle \cap H_{j}\right)=L_{j} \cap \mathcal{L}(\langle\operatorname{Im} f\rangle)$ for all $j \in\{1, \ldots, q\}$, which completes the proof of Theorem 1.2 .

\section{REFERENCES}

1. Z. Chen and Q. Yan, Uniqueness theorem of meromorphic maps into $\mathbb{P}^{N}(\mathbb{C})$ sharing $2 N+3$ hyperplanes regardless of multiplicities, Internat. J. Math. 20 (2009), 717-726. MR2541932 (2010g:32022)

2. G. Dethloff and T. V. Tan, Uniqueness theorems for meromorphic mappings with few hyperplanes, Bulletin des Sciences Mathématiques, 133 (2009), 501-514. MR2538011 (2010g:32023)

3. H. Fujimoto, The uniqueness problem of meromorphic maps into the complex projective space, Nagoya Math. J. 58 (1975), 1-23. MR0393586 (52:14395)

4. H. Fujimoto, Uniqueness problem with truncated multiplicities in value distribution theory, Nagoya Math. J. 152 (1998), 131-152. MR1659377 (99m:32029)

5. S. Ji, Uniqueness problem without multiplicities in value distribution theory, Pacific J. Math. 135 (1988), 323-348. MR968616 (90b:32050)

6. R. Nevanlinna, Einige Eindeutigkeitssätze in der Theorie der meromorphen Funktionen, Acta Math. 48 (1926), 367-391. MR.1555233

7. J. Noguchi, A note on entire pseudo-holomorphic curves and the proof of Cartan-Nochka's theorem, Kodai Math. J. 28 (2005), 336-346. MR2153921 (2006e:32030)

8. S. D. Quang, Unicity problem of meromorphic mappings sharing few hyperplanes, to appear, Ann. Polon. Math.

9. L. Smiley, Geometric conditions for unicity of holomorphic curves, Contemp. Math. 25, Amer. Math. Soc., 1983, 149-154. MR730045 (85j:32042)

10. W. Stoll, On the propagation of dependences, Pacific J. Math. 139 (1989), 311-337. MR.1011216 (91a:32037)

11. D. D. Thai and S. D. Quang, Uniqueness problem with truncated multiplicities of meromorphic mappings in several complex variables, Inter. J. Math. 17 (2006), 1223-1257. MR2287675 (2008f:32022)

Université de Brest, Laboratoire de mathématiques, UMR CNRS 6205, 6, avenue Le Gorgeu, BP 452, 29275 Brest Cedex, France

E-mail address: gerd.dethloff@univ-brest.fr

Hanoi National University of Education, 136-Xuan Thuy Street, Cau Giay, Hanoi, VIETNAM

E-mail address: ducquang.s@gmail.com

Hanoi National University of Education, 136-Xuan Thuy Street, Cau Giay, Hanoi, VIETNAM

E-mail address: tranvantanhn@yahoo.com 